\title{
Limit theorems for fuzzy random variables
}

\author{
By E. P. Klement ${ }^{1} \dagger$, M. L. PuRi ${ }^{2}$ and D. A. Ralescu ${ }^{1}$ \\ ${ }^{1}$ Department of Mathematics, University of Cincinnati, \\ Cincinnati, Ohio 45221, U.S.A. \\ ${ }^{2}$ Department of Mathematics, Indiana University, Bloomington, \\ Indiana 47405, U.S.A.
}

(Communicated by I. N. Sneddon, F.R.S. - Received 7 November 1985)

A strong law of large numbers and a central limit theorem are proved for independent and identically distributed fuzzy random variables, whose values are fuzzy sets with compact levels. The proofs are based on embedding theorems as well as on probability techniques in Banach space.

\section{INTRODUCTION}

The concept of fuzzy set was introduced by Zadeh ( 1965 ). Subsequent developments focused on applications of this concept to pattern recognition and system analysis, among other areas (see Negoita \& Ralescu 1975). Puri \& Raleseu (1986) studied fuzzy random variables as a generalization of random sets. The purpose of this generalization was the introduction of statistical techniques (such as estimation) for pattern recognition. Consider, for example, the problem of recognition of a handwritten character. A random sample is taken from that character. How can the expected character (or a prototype) be defined, and how can it be estimated? The data here are represented by fuzzy sets rather than numbers. The strong law of large numbers (SLLN) and the central limit theorem (CLT) for fuzzy random variables are first steps in the direction of the estimation of vague parameters. After some preliminaries on random sets introduced in $\$ 2$, we introduce various spaces of fuzzy sets and distances on these spaces in $\S 3$. We prove, with respect to one of these distances, that the space of fuzzy sets with compact levels is separable. We also extend the Rådström (1952) embedding theorem to the space of fuzzy sets with compact convex levels. In $\$ 4$ we recall some facts about fuzzy random variables and their expected value. These concepts were defined by Puri \& Ralescu (1986). We prove the Lebesgue dominated convergence type theorem. The result concerning SLLN for fuzzy random variables is given in $\S 5$. This result generalizes the corresponding result for random sets due to Artstein \& Vitale (1975). In $\$ 6$, we prove an embedding theorem for the space of fuzzy sets with compact convex levels satisfying a Lipschitz condition. This embedding is a key tool in proving our CLT for fuzzy random variables in $\S 7$. Our results extends the

† Permanent address: Institut für Mathematik, Johannes Kepler Universität, A-4040 Linz, Austria. 
CLT for random sets (ef. Weil 1982; Giné et al. 1982). Finally, in §8, we comment on some spaces of fuzzy sets where the CLT can be applied.

Our approach relies heavily on probability techniques in Banach space. As with random sets, our SLLN is not an immediate application of those techniques, since the spaces of fuzzy sets are not Banach spaces. In fact, they are not even vector spaces, so we rely on embedding theorems as mentioned in $\$ \S 3$ and 6 . Our framework is more complex than that of random sets: on the one hand, separability and therefore measurability problems arise; on the other hand, since an integral similar to that of Debreu ( 1967 ) is not available for fuzzy random variables, our proof of the SLLN uses a truncation argument and a Lebesgue dominated convergence type theorem. Also, due to the complexity of spaces of fuzzy sets (as compared to spaces of sets), our CLT has a more complicated form than the CLT for random sets.

\section{Preliminaries on Random Sets}

Let $\mathscr{K}\left(\mathbb{R}^{p}\right)$ denote the collection of non-empty compact subsets of the Euclidean space $\mathbb{R}^{p}$, and let $\mathscr{K}_{\mathrm{c}}\left(\mathbb{R}^{p}\right)$ denote the non-empty compact convex subsets of $\mathbb{R}^{p}$. The space $\mathscr{K}\left(\mathbb{R}^{p}\right)$ has a linear structure induced by the (Minkowski) addition and scalar multiplication:

$$
A+B=\{a+b \mid a \in A, b \in B\}, \lambda A=\{\lambda a \mid a \in A\}
$$

for $A, B \in \mathscr{K}\left(\mathbb{R}^{p}\right), \lambda \in \mathbb{R}$. However, $\mathscr{K}\left(\mathbb{R}^{p}\right)$ is not a vector space (it is not a group with respect to addition).

The space $\mathscr{K}\left(\mathbb{R}^{p}\right)$ is metrizable by the Hausdorff distance

$$
d(A, B)=\max \left\{\sup _{a \in A} \inf _{b \in B}\|a-b\|, \sup _{b \in B} \inf _{a \in A}\|a-b\|\right\}
$$

where \|\| denotes the euclidean norm and $A, B \in \mathscr{K}\left(\mathbb{R}^{p}\right)$. It is well known that $\left(\mathscr{K}\left(\mathbb{R}^{p}\right), d\right)$ is a complete separable metric space (Debreu (i 967$)$ ). If $A \in \mathscr{K}\left(\mathbb{R}^{p}\right)$, we write $\|A\|=d(A,\{0\})$.

Let $(\Omega, \mathscr{A}, P)$ be a probability space. A random set is a Borel measurable function $f: \Omega \rightarrow \mathscr{K}\left(\mathbb{R}^{p}\right)$.

The expected value $E f$ of a random set was defined by Aumann (1965) as:

$$
E f=\left\{E \phi \mid \phi \in L^{1}(\Omega, \mathscr{A}, P), \quad \phi(\omega) \in f(\omega) \text { a.e. }\right\}
$$

where $\phi: \Omega \rightarrow \mathbb{R}^{p}$ is a selection of $f$ and $E \phi$ denotes the expectation of the random vector $\phi$. If $f$ is $\mathscr{K}_{\mathrm{c}}\left(\mathbb{R}^{p}\right)$-valued and if $E\|f\|<\infty$, then $E f \in \mathscr{K}_{\mathrm{c}}\left(\mathbb{R}^{p}\right)$.

Various properties related to the calculus of set-valued functions (including the Lebesgue dominated convergence type theorems) are discussed in Aumann (1965) and Debreu ( 1967$)$. A comprehensive theory of random sets is given in Matheron (1975). 


\section{SPACES OF FUZZY SETS}

A fuzzy subset of $\mathbb{R}^{p}($ fuzzy set $)$ is a function of $u: \mathbb{R}^{p} \rightarrow[0,1]$. For each such fuzzy set $u$, we denote by $L_{\alpha} u=\left\{x \in \mathbb{R}^{p} \mid u(x) \geqslant \alpha\right\}, 0 \leqslant \alpha \leqslant 1$, its $\alpha$-level set. By $\operatorname{supp} u$ we denote the support of $u$, i.e. the closure of the set $\left\{x \in \mathbb{R}^{p} \mid u(x)>0\right\}$.

We consider the collection $\mathscr{F}\left(\mathbb{R}^{p}\right)$ of those fuzzy sets $u: \mathbb{R}^{p} \rightarrow[0,1]$ with the following properties.

(1) $u$ is upper semicontinuous; (2) supp $u$ is compact; (3) $\left\{x \in \mathbb{R}^{p} \mid u(x)=1\right\} \neq \varnothing$.

The space $\mathscr{F}\left(\mathbb{R}^{p}\right)$ extends $\mathscr{K}\left(\mathbb{R}^{p}\right)$ in the sense that for each $A \in \mathscr{K}\left(\mathbb{R}^{p}\right)$, its characteristic function $\chi_{A} \in \mathscr{F}\left(\mathbb{R}^{p}\right)$.

A linear structure in $\mathscr{F}\left(\mathbb{R}^{p}\right)$ is defined via the following operations:

$$
\begin{array}{r}
(u+v)(x)=\sup _{y+z-x} \min [u(y), v(z)], \\
(\lambda u)(x)=\left\{\begin{array}{l}
u\left(\lambda^{-1} x\right) \text { if } \lambda \neq 0 \\
\chi_{\{0\}}(x) \text { if } \lambda=0
\end{array}\right.
\end{array}
$$

for $u, v \in \mathscr{F}\left(\mathbb{R}^{p}\right), \lambda \in \mathbb{R}$.

By using simple topological arguments and properties of upper semicontinuous functions, it is easy to see that $u+v, \lambda u \in \mathscr{F}\left(\mathbb{R}^{p}\right)$. The following properties will be needed later:

$$
L_{\alpha}(u+v)=L_{\alpha} u+L_{\alpha} v \text { and } L_{\alpha}(\lambda u)=\lambda L_{\alpha} u
$$

for every $0 \leqslant \alpha \leqslant 1$.

There is no unique metric in $\mathscr{F}\left(\mathbb{R}^{p}\right)$ which extends the Hausdorff distance.

In this paper we will mainly be concerned with the metric defined as

$$
d_{1}(u, v)=\int_{0}^{1} d\left(L_{\alpha} u, L_{\alpha} v\right) \mathrm{d} \alpha .
$$

We will also use the metric

$$
d_{\infty}(u, v)=\sup _{\alpha>0} d\left(L_{\alpha} u, L_{\alpha} v\right)
$$

which was studied in Puri \& Raleseu $(1983 b)$

Clearly, if $A, B \in \mathscr{K}\left(\mathbb{R}^{p}\right)$, then

$$
d_{1}\left(\chi_{A}, \chi_{B}\right)=d_{\infty}\left(\chi_{A}, \chi_{B}\right)=d(A, B) .
$$

Proposition 3.1. $\left(\mathscr{F}\left(\mathbb{R}^{p}\right), d_{1}\right)$ is a metric space.

Proof: Obviously $d_{1}(u, v)<\infty$ since $\operatorname{supp} u$ and $\operatorname{supp} v$ are bounded.

The function $\phi(\alpha)=d\left(L_{\alpha} u, L_{\alpha} v\right)$ is measurable: if $\alpha_{1} \leqslant \alpha_{2} \leqslant \ldots$ with $\lim \alpha_{n}=\alpha$, then $L_{\alpha} u=\bigcap_{n=1}^{\infty} L_{\alpha_{n}} u, \quad L_{\alpha} v=\bigcap_{n=1}^{\infty} L_{\alpha_{n}} v$. Thus $d\left(L_{\alpha_{n}} u, L_{\alpha} u\right) \rightarrow 0, \quad d\left(L_{\alpha_{n}} v\right.$, $\left.L_{\alpha} v\right) \rightarrow 0$ and, from the continuity of the Hausdorff distance, we conclude that $\phi$ is left-continuous and therefore measurable.

The triangle inequality and symmetry property of $d_{1}$ are clear. It remains to show that $d_{1}(u, v)=0$ implies $u=v$.

If $d_{1}(u, v)=0$, it follows that $L_{\alpha} u=L_{\alpha} v$ a.e., therefore $u$ and $v$ have equal levels for $\alpha \in[0,1] \backslash A$ where $\lambda(A)=0$ ( $\lambda$ denotes the Lebesgue measure).

It is well known that $[0,1] \backslash A$ is dense in $[0,1]$. If $\alpha_{0} \in A, \alpha_{0}>0$, there exists an 
increasing sequence $\alpha_{n} \in[0,1] \backslash A, \alpha_{n} \rightarrow \alpha_{0}$. Then

$$
L_{\alpha_{0}} u=\bigcap_{n=1}^{\infty} L_{\alpha_{n}} u=\bigcap_{n=1}^{\infty} L_{\alpha_{n}} v=L_{\alpha_{0}} v .
$$

So $L_{\alpha} u=L_{\alpha} v$ for each $\alpha \in[0,1]$, which implies $u=v$.

A crucial property in connection with the strong law of large numbers is that of separability (see, e.g. Taylor ${ }_{1978}$ ). It turns out that the space $\left(\mathscr{F}\left(\mathbb{R}^{p}\right), d_{1}\right)$ is separable. The space $\left(\mathscr{F}\left(\mathbb{R}^{p}\right), d_{\infty}\right)$, on the other hand, is not separable, which means that the distance $d_{1}$ is preferable to $d_{\infty}$. These statements are proved in the following.

Proposition 3.2. The metric space $\left(\mathscr{F}\left(\mathbb{R}^{p}\right), d_{1}\right)$ is separable.

Proof. Suppose that $u \in \mathscr{F}\left(\mathbb{R}^{p}\right)$ and $\epsilon>0$ are given.

1. Since $\operatorname{supp} u$ is compact, there exist $p$-dimensional cubes $S_{i}=\prod_{j-1}^{p}\left[a_{i j}, b_{i j}\right)$, $i=1, \ldots, r$ such that $a_{i j}, b_{i j} \in \mathbb{Q}, 0<b_{i j}-a_{i j} \leqslant(2 \epsilon) / \sqrt{ } p$, and $\operatorname{supp} u \subseteq \bigcup_{i=1}^{r} S_{i}$. Consider the corner point $T_{i}=\left(a_{i 1}, \ldots, a_{i p}\right)$ of $S_{i}$ and define the fuzzy set $\phi$ : $\mathbb{R}^{p} \rightarrow[0,1]$ by

$$
\phi(x)=\left\{\begin{array}{l}
\operatorname{supp}_{x \in \bar{S}_{i}} u(x) \quad \text { if } x=T_{i}, i=1, \ldots, r, \\
0 \quad \text { otherwise. }
\end{array}\right.
$$

Obviously we have $\phi \in \mathscr{F}\left(\mathbb{R}^{p}\right)$. Putting $\alpha_{i}=\phi\left(T_{i}\right)$, we relabel $S_{1}, \ldots, S_{r}$ and $T_{1}, \ldots, T_{r}$ (if necessary) such that $0=\alpha_{0} \leqslant \alpha_{1} \leqslant \alpha_{2} \leqslant \ldots \leqslant \alpha_{r}=1$.

2. We claim that $d_{\infty}(u, \phi) \leqslant \frac{1}{2} \epsilon$. To this end we choose an $\alpha>0$. Since $\alpha_{i_{0}-1}<\alpha \leqslant \alpha_{i_{0}}$ for some $1 \leqslant i_{0} \leqslant r$ we get $L_{\alpha} u \supseteq L_{\alpha_{i_{0}}} u$ and $L_{\alpha} \phi=L_{\alpha_{i_{0}}} \phi=$ $\left\{T_{i_{0}}, T_{i_{0}+1}, \ldots, T_{r}\right\}$. As $x \in L_{\alpha} u$ implies $x \in S_{i_{1}}$ for some $i_{1} \geqslant i_{0}$, it follows that $\min _{i_{0} \leqslant i \leqslant r}\left\|x-T_{i}\right\| \leqslant\left\|x-T_{i_{1}}\right\| \leqslant \frac{1}{2} \epsilon$.

On the other hand, for any $i \geqslant i_{0}$, we have $\phi\left(T_{i}\right)=\operatorname{supp}_{x \in S_{i}} u(x) \geqslant \alpha$. Since $u$ is upper semicontinuous, it attains its supremum at some point $x_{0} \in \bar{S}_{i} \cap L_{\alpha} u$. This gives

Therefore

$$
\inf _{x \in L_{\alpha} u}\left\|T_{i}-x\right\| \leqslant\left\|T_{i}-x_{0}\right\| \geqslant \frac{1}{2} \epsilon .
$$

$$
d_{\infty}(u, \phi)=\sup _{\alpha>0} d\left(L_{\alpha} u, L_{\alpha} \phi\right)=\sup _{\alpha>0} d\left(L_{\alpha} u,\left\{T_{i_{0}}, \ldots, T_{r}\right\}\right) \leqslant \frac{1}{2} \epsilon .
$$

3. If necessary, we relabel $\alpha_{1}, \ldots, \alpha_{r}$ to obtain $0 \leqslant \alpha_{1}<\alpha_{2}<\ldots<\alpha_{s}=1$ with $s \leqslant r$. If $\alpha_{k} \notin \mathbb{Q}$, we choose $\beta_{k} \in \mathbb{Q}$ such that $\max \left(\alpha_{k-1}, \alpha_{k}-\epsilon / M\right)<\beta_{k}<\alpha_{k}$ with $M>2(r-1) \operatorname{diam}(\operatorname{supp} u)$, while if $\alpha_{k} \in \mathbb{Q}$, we set $\beta_{k}=\alpha_{k}(k=1, \ldots, s)$. Here $\operatorname{diam}(\operatorname{supp} u)$ stands for the diameter of $\operatorname{supp} u$.

Defining $\psi \in \mathscr{F}\left(\mathbb{R}^{p}\right)$ by

$$
\psi(x)=\left\{\begin{array}{l}
\beta_{k} \text { if } \phi(x)=\alpha_{k} \\
0 \text { otherwise }
\end{array}\right.
$$

yields

$$
\begin{aligned}
d_{1}(\phi, \psi) & =\int_{0}^{1} d\left(L_{\alpha} \phi, L_{\alpha} \psi\right) \mathrm{d} \alpha=\sum_{i=1}^{s-1} \int_{\beta_{i}}^{\alpha_{i}} d\left(L_{\alpha} \phi, L_{\alpha} \psi\right) \mathrm{d} \alpha \\
& \leqslant \operatorname{diam}(\operatorname{supp} u) \cdot \sum_{i=1}^{s-1}\left(\alpha_{i}-\beta_{i}\right)<\frac{1}{2} \epsilon .
\end{aligned}
$$


4. By the triangle inequality, we get

$$
d_{1}(u, \psi) \leqslant d_{1}(u, \phi)+d_{1}(\phi, \psi) \leqslant d_{\infty}(u, \phi)+d_{1}(\phi, \psi)<\epsilon .
$$

Since the set of functions $\psi$ is countable, separability of $\left(\mathscr{F}\left(\mathbb{R}^{p}\right), d_{1}\right)$ follows.

Proposition $3.3\left(\mathscr{F}\left(\mathbb{R}^{p}\right), d_{\infty}\right)$ is not separable.

Proof. Put $a=(0,0, \ldots, 0) \in \mathbb{R}^{p}$ and $b=(1,0,0, \ldots, 0) \in \mathbb{R}^{p}$. For each $\alpha \in(0,1)$, define $\phi_{\alpha} \in \mathscr{F}\left(\mathbb{R}^{p}\right)$ by

$$
\phi_{\alpha}(x)= \begin{cases}1 & \text { if } x=b \\ \alpha & \text { if } x \in \cos \{a, b\} \backslash\{b\} \\ 0 & \text { otherwise }\end{cases}
$$

where co $\{a, b\}$ is the convex hull of $\{a, b\}$.

It is easy to check that $d_{\infty}\left(\phi_{\alpha}, \phi_{\beta}\right)=1$ for $\alpha \neq \beta$.

Denote by $\mathscr{F}_{\mathrm{c}}\left(\mathbb{R}^{p}\right)$ the space of fuzzy sets $u \in \mathscr{F}\left(\mathbb{R}^{p}\right)$ such that $L_{\alpha} u$ is convex for each $\alpha \geqslant 0$. Alternatively, $u \in \mathscr{F}_{\mathrm{c}}\left(\mathbb{R}^{p}\right)$ if $u \in \mathscr{F}\left(\mathbb{R}^{p}\right)$ and $u$ is fuzzy convex (i.e. $u(\lambda x+(1-\lambda) y) \geqslant \min [u(x), u(y)]$ for all $x, y \in \mathbb{R}^{p}, \lambda \in[0,1]$; see Zadeh (I965)).

The space $\mathscr{F}_{\mathrm{e}}\left(\mathbb{R}^{p}\right)$ plays an important role since it can be embedded isometrically into a Banach space. Actually this embedding generalizes the Rådström embedding theorem (Rådström I 952) of $\mathscr{K}_{\mathrm{c}}\left(\mathbb{R}^{p}\right)$ into a Banach space.

Such an embedding for fuzzy sets is given in Puri \& Ralescu $(1983 b)$, in a more general framework. For completeness we state this result here and sketch its proof.

Proposition 3.4. There exists a normed space $\chi$ and a function $j: \mathscr{F}_{\mathrm{c}}\left(\mathbb{R}^{p}\right) \rightarrow \chi$ with properties:

(a) $j$ is an isometry $\left(\right.$ i.e. $\left.\|j(u)-j(v)\|=d_{1}(u, v)\right)$,

(b) $j(u+v)=j(u)+j(v)$,

(c) $j(\lambda u)=\lambda j(u), \lambda \geqslant 0$.

Proof. Define an equivalence relation in $\mathscr{F}_{\mathrm{c}}\left(\mathbb{R}^{p}\right) \times \mathscr{F}_{\mathrm{c}}\left(\mathbb{R}^{p}\right)$ by

$$
(u, v) \sim\left(u^{\prime}, v^{\prime}\right) \Leftrightarrow u+v^{\prime}=u^{\prime}+v .
$$

A general theorem of Rådström (1952) can be used; the necessary hypotheses are verified as in Puri \& Ralescu ( $1983 b)$. Ths space of equivalence classes $[u, v]$ of pairs $(u, v)$ is denoted by $\chi$. The norm in $\chi$ is defined by $\|[u, v]\|=d_{1}(u, v)$. It is easy to check that $j: \mathscr{F}_{\mathrm{e}}\left(\mathbb{R}^{p}\right) \rightarrow \chi$ defined by $j(u)=\left[u, \chi_{\{0\}}\right]$ is an isometry, i.e. $\|j(u)-j(v)\|=d_{1}(u, v)$, and properties $(b),(c)$ follow from the definitions.

\section{FUZZY RANDOM VARIABLES}

The concept of a random set was generalized by Puri \& Ralescu (1986). A fuzzy random variable (FRV) is a Borel measurable function $X: \Omega \rightarrow\left(\mathscr{F}\left(\mathbb{R}^{p}\right), d_{\infty}\right)$. Note that we use $d_{\infty}$ here and not $d_{1}$; the reason for this will soon become clear.

If $X$ is a fuzzy random variable such that $E\|\operatorname{supp} X\|<\infty$, then the expected value $E X$ is the (unique) fuzzy set satisfying the property

$$
L_{\alpha}(E X)=E\left(L_{\alpha} X\right), 0<\alpha \leqslant 1
$$

(Puri \& Ralescu 1986, Theorem 3.1). 
It follows that $E X: \mathbb{R}^{p} \rightarrow[0,1]$ is upper semicontinuous and $L_{1}(E X) \neq \varnothing$.

Also supp $E X$ is compact; to see this, note that it suffices to show $L_{\alpha}(E X) \subseteq K$ for every $\alpha>0$ and for some compact set $K$. But $L_{\alpha}(E X)=E\left(L_{\alpha} X\right) \subseteq E(\operatorname{supp} X)$. Since $E\|\operatorname{supp} X\|<\infty$, it follows that $E(\operatorname{supp} X)$ is a non-empty compact set, so we can take $K=E(\operatorname{supp} X)$.

In the discussion above, it is implicit that $\operatorname{supp} X$ is measurable, i.e. it is a random set. This fact follows from the following.

Proposition 4.1. If $X$ is a fuzzy random variable, then $\operatorname{supp} X$ is a random set.

Proof. It is enough to show that the function $\phi:\left(\mathscr{F}\left(\mathbb{R}^{p}\right), d_{\infty}\right) \rightarrow$ $\left(\mathscr{K}\left(\mathbb{R}^{p}\right), d\right), \phi(u)=\operatorname{supp} u$ is Borel measurable. Note that $d\left(L_{1 / n} u, \operatorname{supp} u\right) \rightarrow 0$ as $n \rightarrow \infty$. So, if we define $\phi_{n}(u)=L_{1 / n} u$, then $\phi=\lim _{n \rightarrow \infty} \phi_{n}$ pointwise. Now $\phi_{n}$ are obviously continuous functions, so $\phi$ is measurable.

Note. A similar argument will not hold if $d_{\infty}$ is replaced by $d_{1}$.

From the above result, it follows that if $X$ is a fuzzy random variable, then $E X \in \mathscr{F}\left(\mathbb{R}^{p}\right)$.

An important property of the expected value, which will be needed later, is the Lebesgue dominated convergence type theorem. Such a result is contained in Puri $\&$ Ralescu (1986), but for the metric $d_{\infty}$.

We state and prove the new version here, in the form that we need in the next section. By $\{0\}$ we denote the one point set containing 0 (identified with its characteristic function $\left.\chi_{\{0\}}\right)$.

Theorem 4.1. Let $\left\{X_{k} \mid k \geqslant 1\right\}, X$ be fuzzy random variables with values in $\mathscr{F}_{c}\left(\mathbb{R}^{p}\right)$ and such that $E\left\|\operatorname{supp} X_{k}\right\|<\infty, E\|\operatorname{supp} X\|<\infty$. Suppose that $X_{k} \rightarrow X$ a.e. in the metric $d_{1}$, and that $d_{1}\left(X_{k}(\omega),\{0\}\right) \leqslant h(\omega)$ for all $k \geqslant 1$, where $h: \Omega \rightarrow \mathbb{R}$ is integrable. Then $E X_{k} \rightarrow E X$ in the metric $d_{1}$.

Proof.

$$
\begin{aligned}
d_{1}\left(E X_{k}, E X\right) & =\int_{0}^{1} d\left[L_{\alpha}\left(E X_{k}\right), L_{\alpha}(E X)\right] \mathrm{d} \alpha \\
& =\int_{0}^{1} d\left[E\left(L_{\alpha} X_{k}\right), E\left(L_{\alpha} X\right)\right] \mathrm{d} \alpha \leqslant \int_{0}^{1} E\left[d\left(L_{\alpha} X_{k}, L_{\alpha} X\right)\right] \mathrm{d} \alpha
\end{aligned}
$$

by using an inequality of Debreu (1967, p. 366-367). From the Fubini theorem we conclude that

$$
d_{1}\left(E X_{k}, E X\right) \leqslant E\left[d_{1}\left(X_{k}, X\right)\right] .
$$

From the hypothesis, $d_{1}\left(X_{k}, X\right) \rightarrow 0$ a.e. Also,

$$
d_{1}\left(X_{k}, X\right) \leqslant d_{1}\left(X_{k},\{0\}\right)+d_{1}(\{0\}, X) \leqslant h+d_{1}(\{0\}, X) .
$$

From the classical Lebesgue dominated convergence theorem, it follows that $d_{1}\left(E X_{k}, E X\right) \rightarrow 0$, concluding the proof.

\section{LAW OF LARGE NUMBERS}

The strong law of large numbers (SLLN) for random sets was derived by Artstein $\&$ Vitale (1975). This was extended to random convex sets in a Banach space by Puri \& Ralescu (1983a, 1985). 
Here we generalize the Artstein-Vitale result to fuzzy random variables. Note that such a SLLN was stated by Féron (1979) by using a different metric, but no proof was provided.

We will consider fuzzy random variables with values in $\mathscr{F}\left(\mathbb{R}^{p}\right)$. Note that it makes sense to talk about independent and identically distributed FRVs, since $\left(\mathscr{F}\left(\mathbb{R}^{p}\right), d_{\infty}\right)$ is a metric space (see Billingsley 1968 ).

We will also need the concept of a convex hull of a fuzzy set (see Lowen 1980).

If $u \in \mathscr{F}\left(\mathbb{R}^{p}\right)$, then co $u \in \mathscr{F}_{\mathrm{c}}\left(\mathbb{R}^{p}\right)$, the convex hull of $u$ is defined by

$$
\operatorname{co} u=\inf \left\{v \in \mathscr{F}_{\mathrm{c}}\left(\mathbb{R}^{p}\right) \mid v \geqslant u\right\} .
$$

It is possible to show that

$$
L_{\alpha}(\cos u)=\operatorname{co}\left(L_{\alpha} u\right), 0 \leqslant \alpha \leqslant 1 .
$$

If $X: \Omega \rightarrow \mathscr{F}\left(\mathbb{R}^{p}\right)$ is a fuzzy random variable, then co $X: \Omega \rightarrow \mathscr{F}_{\mathrm{c}}\left(\mathbb{R}^{p}\right)$ is defined by $(\operatorname{co} X)(\omega)=\operatorname{co} X(\omega)$.

Our main result is as follows.

Theorem 5.1. Let $\left\{X_{k} \mid k \geqslant 1\right\}$ be independent and identically distributed fuzzy random variables such that $E\left\|\operatorname{supp} X_{1}\right\|<\infty$. Then

$$
\left(X_{1}+X_{2}+\ldots+X_{n}\right) / n \rightarrow E\left(\operatorname{co} X_{1}\right) \text { a.e., }
$$

the convergence being in the metric $d_{1}$.

Proof. (1) Consider first $X_{k}: \Omega \rightarrow \mathscr{F}_{\mathrm{c}}\left(\mathbb{R}^{p}\right)$ and let $j: \mathscr{F}_{\mathrm{c}}\left(\mathbb{R}^{p}\right) \rightarrow \chi$ be the isometry provided by Proposition 3.4.

Since $\left(\mathscr{F}_{\mathrm{c}}\left(\mathbb{R}^{p}\right), d_{1}\right)$ is separable, it is easy to show that $\chi$ is separable. Then $\left\{j \circ X_{k} \mid k \geqslant 1\right\}$ are IID $\chi$-valued random elements. By a standard SLLN in Banach space, it follows that $1 / n \sum_{k=1}^{n}\left(j \circ X_{k}\right) \rightarrow E\left(j \circ X_{1}\right)$ a.e.

The main point now is to show that $E\left(j \circ X_{1}\right)=j\left(E X_{1}\right)$ if $E\left\|\operatorname{supp} X_{1}\right\|<\infty$.

Assume first that $X_{1}$ is a simple function, i.e. $X_{1}=\sum_{i=1}^{l} u_{i} \chi_{A_{i}}$, $u_{i} \in \mathscr{F}_{\mathrm{c}}\left(\mathbb{R}^{p}\right), A_{i} \in \mathscr{A}$. It is easy to check that $E\left(j \circ X_{1}\right)=j\left(E X_{1}\right)$ in this case.

Since $X_{1}$ is measurable, there exists a sequence of simple functions (FRvs) $s_{\mathrm{m}}$ with $s_{\mathrm{m}} \rightarrow X_{1}$ a.e. in the metric $d_{1}$ (note that we can not assert the existence of such a sequence which converges in $d_{\infty}$, unless $X_{1}$ is separably valued).

Also $d_{1}\left(s_{\mathrm{m}},\{0\}\right) \rightarrow d_{1}\left(X_{1},\{0\}\right)$ a.e. from the continuity of $d_{1}$. Consider the truncated FRVs $t_{m}$ as follows:

$$
t_{m}(\omega)=\left\{\begin{array}{l}
s_{\mathrm{m}}(\omega), \text { if } d_{1}\left(s_{\mathrm{m}}(\omega),\{0\}\right) \leqslant 2 d_{1}\left(X_{1}(\omega),\{0\}\right) \\
\{0\}, \quad \text { otherwise. }
\end{array}\right.
$$

Note that $t_{m}$ are simple functions. It is easy to see that $d_{1}\left(t_{m}, X_{1}\right) \rightarrow 0$ a.e. and that $d_{1}\left(t_{m}(\omega),\{0\}\right) \leqslant 2 d_{1}\left(X_{1}(\omega),\{0\}\right)$.

The hypotheses of Theorem 4.1 are satisfied, so $d_{1}\left(E t_{m}, E X_{1}\right) \rightarrow 0$. Therefore $j\left(E t_{m}\right) \rightarrow j\left(E X_{1}\right)$ in $\chi$. It is easy to see that $j \circ t_{m} \rightarrow j \circ X_{1}$ and, from properties of the Bochner integral, that $E\left(j \circ t_{m}\right) \rightarrow E\left(j \circ X_{1}\right)$. Since $j\left(E t_{m}\right)=E\left(j \circ t_{m}\right)$ it follows that $j\left(E X_{1}\right)=E\left(j \circ X_{1}\right)$.

Therefore $\left\|1 / n \sum_{k=1}^{n}\left(j \circ X_{k}\right)-j\left(E X_{1}\right)\right\| \rightarrow 0$ a.e.

From the properties of $j$, it follows that $d_{1}\left(1 / n \sum_{k-1}^{n} X_{k}, E X_{1}\right) \rightarrow 0$ a.e. 
Consider now the general case, i.e. $X_{k}: \Omega \rightarrow \mathscr{F}\left(\mathbb{R}^{p}\right)$. Since $\left\{\right.$ co $\left.X_{k} \mid k \geqslant 1\right\}$ are IID, and $E\left\|\operatorname{supp}\left(\operatorname{co} X_{1}\right)\right\| \leqslant E\left\|\operatorname{supp} X_{1}\right\|<\infty$, it follows from part (1) that

$$
\left(\sum_{k=1}^{n} \operatorname{co} X_{k}\right) / n \stackrel{d_{1}}{\rightarrow} E\left(\operatorname{co} X_{1}\right)
$$

The Shapley-Folkman lemma (see Arrow \& Hahn 1971) gives;

$$
d\left[L_{\alpha}\left(\frac{1}{n} \sum_{k=1}^{n} X_{k}\right), L_{\alpha}\left(\frac{1}{n} \sum_{k=1}^{n} \operatorname{co} X_{k}\right)\right] \leqslant \frac{\sqrt{ } p}{n} \max _{1 \leqslant k \leqslant n}\left\|L_{\alpha} X_{k}\right\|,
$$

for every $\alpha>0$. This implies immediately

$$
d_{\infty}\left(\frac{1}{n} \sum_{k=1}^{n} X_{k}, \frac{1}{n} \sum_{k=1}^{n} \operatorname{co} X_{k}\right) \leqslant \frac{\sqrt{ } p}{n} \max _{1 \leqslant k \leqslant n}\left\|\operatorname{supp} X_{k}\right\| .
$$

Since $(1 / n) \max _{1 \leqslant k \leqslant n}\left\|\operatorname{supp} X_{k}\right\| \rightarrow 0$ a.e. (see, for example, Chow \& Teicher 1978 , p. 122), it follows that $d_{1}\left((1 / n) \sum_{k=1}^{n} X_{k},(1 / n) \sum_{k=1}^{n}\right.$ co $\left.X_{k}\right) \rightarrow 0$ a.e. Finally, from the triangle inequality, it follows that $d_{1}\left[(1 / n) \sum_{k=1}^{n} X_{k}, E\left(\operatorname{co} X_{1}\right)\right] \rightarrow 0$ a.e. and the proof is complete.

Note. From the above proof it seems that we could prove the SLLN with respect to $d_{\infty}$, at least for $\mathscr{F}_{\mathrm{c}}\left(\mathbb{R}^{p}\right)$-valued fuzzy random variables. This is not the case since $\left(\mathscr{F}_{\mathrm{c}}\left(\mathbb{R}^{p}\right), d_{\infty}\right)$ is not separable. This follows from Proposition 3.3 (the same example works).

\section{THE EMBEDDING THEOREM}

Let $S^{p-1}=\left\{x \in \mathbb{R}^{p} \mid\|x\|=1\right\}$ denote the unit sphere in $\mathbb{R}^{p}$. It is well known that $\mathscr{K}_{\mathrm{c}}\left(\mathbb{R}^{p}\right)$ can be embedded isometrically into $C\left(S^{p-1}\right)$ (the Banach space of continuous functions on $S^{p-1}$ ). This result goes back to Minkowski (see, for example, Artstein \& Vitale 1975).

Such an embedding is realized via the support function of a compact convex set. More precisely, if $K \in \mathscr{K}_{\mathrm{c}}\left(\mathbb{R}^{p}\right)$, its support function is defined by

$$
s_{K}(x)=\sup _{a \in K}\langle x, a\rangle, \quad x \in S^{p-1} .
$$

In the case of fuzzy sets, more restrictions should be imposed in order to achieve a similar embedding.

Consider the space $\mathscr{F}_{L}\left(\mathbb{R}^{p}\right)$ of fuzzy sets $u \in \mathscr{F}\left(\mathbb{R}^{p}\right)$ such that the map $\alpha \rightarrow L_{\alpha} u$ is Lipschitz. More specifically, $u \in \mathscr{F}_{L}\left(\mathbb{R}^{p}\right)$ if there exists a constant $M>0$, such that

$$
d\left(L_{\alpha} u, L_{\beta} u\right) \leqslant M|\alpha-\beta|
$$

for every $\alpha, \beta \in(0,1]$.

Define the space $\mathscr{F}_{C L}\left(\mathbb{R}^{p}\right)=\mathscr{F}_{L}\left(\mathbb{R}^{p}\right) \cap \mathscr{F}_{\mathrm{c}}\left(\mathbb{R}^{p}\right)$. The next theorem gives the desired embedding.

THEOREM 6.1 There exists a function

$$
j: \mathscr{F}_{C L}\left(\mathbb{R}^{p}\right) \rightarrow C\left([0,1] \times S^{p-1}\right)
$$


such that:

(a) $j$ is an isometry $\left(i . e .\|j(u)-j(v)\|_{\infty}=d_{\infty}(u, v)\right)$,

(b) $j(u+v)=j(u)+j(v)$,

(c) $j(\lambda u)=\lambda j(u), \lambda \geqslant 0$.

Proof. Define $j(u)=s_{u}$, where

$$
s_{u}(\alpha, x)=\left\{\begin{array}{l}
{ }_{L_{\alpha} u}(x), \text { if } \alpha>0 \\
s_{\operatorname{supp} u}(x), \text { if } \alpha=0 .
\end{array}\right.
$$

The function $s_{u}$ is Lipschitz on $[0,1] \times S^{p-1}$ :

$$
\begin{aligned}
\left|s_{u}(\alpha, x)-s_{u}(\beta, y)\right| & \leqslant\left|s_{L_{\alpha} u}(x)-s_{L_{\beta} u}(x)\right|+\left|s_{L_{\beta} u}(x)-s_{L_{\beta} u}(y)\right| \\
& \leqslant\left\|s_{L_{\alpha} u}-s_{L_{\beta} u}\right\|_{\infty}+\left\|L_{\beta} u\right\|\|x-y\| \\
& \leqslant\|u\|_{L}(|\alpha-\beta|+\|x-y\|)
\end{aligned}
$$

where

$$
\|u\|_{L}=\sup _{\alpha \neq \beta} \frac{d\left(L_{\alpha} u, L_{\beta} u\right)}{|\alpha-\beta|}+\sup _{\alpha>0}\left\|L_{\alpha} u\right\|
$$

and we have used the fact that $K \in \mathscr{K}_{\mathrm{c}}\left(\mathbb{R}^{p}\right) \rightarrow s_{K}$ is an isometry, as well as the fact that $s_{K}$ satisfies a Lipschitz condition with constant $\|K\|$.

Since any two norms in $\mathbb{R}^{p+1}$ are equivalent, inequality (6.1) becomes

$$
\left|s_{u}(\alpha, x)-s_{u}(\beta, y)\right| \leqslant q\|u\|_{L}\left(|\alpha-\beta|^{2}+\|x-y\|^{2}\right)^{\frac{1}{2}},
$$

where $q$ is a constant.

Thus $s_{u}=j(u) \in C\left([0,1] \times S^{p-1}\right)$.

It is now easy to show that $j$ is an isometry, i.e.

$$
\|j(u)-j(v)\|_{\infty}=\sup _{\alpha, x}\left|s_{u}(\alpha, x)-s_{v}(\alpha, x)\right|=d_{\infty}(u, v)
$$

as well as properties $(b)$ and $(c)$, and the proof is completed.

\section{Central Limit theOREM}

The CLT for random sets was first given, in a particular case, by Cressie (r979). The general theorem first appeared in Weil (1982) and, independently, in Giné et al. (I982); Vitale (I98I); Trader \& Eddy (I98I); and Puri \& Ralescu (I985). Lyashenko (1979) even considered the non IID case, although in a metric different from the Hausdorff metric.

We will consider here fuzzy random variables with values in the space $\left(\mathscr{F}_{L}\left(\mathbb{R}^{p}\right), d_{\infty}\right)$. Our main result is as follows.

THEOREM 7.1. $X_{1}, X_{2}, \ldots: \Omega \rightarrow \mathscr{F}_{L}\left(\mathbb{R}^{p}\right)$ be independent and identically distributed fuzzy random variables, satisfying:

(a) $E\left\|\operatorname{supp} X_{1}\right\|^{2}<\infty$,

(b) $E\left[\sup _{\alpha \neq \beta} \frac{d\left(L_{\alpha} X_{1}, L_{\beta} X_{1}\right)}{|\alpha-\beta|}\right]^{2}<\infty$.

Then there exists a Gaussian random element $Z$ in $C\left([0,1] \times S^{p-1}\right)$, such that $\sqrt{ } n d_{\infty}\left(X_{1}+X_{2}+\ldots+X_{n} / n, E\left(\operatorname{co} X_{1}\right)\right) \rightarrow\|Z\|_{\infty}$ weakly. 
Proof. (1) Consider first the convex case, i.e. $X_{n}: \Omega \rightarrow \mathscr{F}_{C L}\left(\mathbb{R}^{p}\right)$.

Let $j: \mathscr{F}_{C L}\left(\mathbb{R}^{p}\right) \rightarrow C\left([0,1] \times S^{p-1}\right)$ be the isometry given by Theorem 6.1 , and consider $Y_{n}=j\left(X_{n}\right)$ as random elements in $C\left([0,1] \times S^{p-1}\right)$.

Observe that inequality (6.2) in the proof of Theorem 6.1 can be rewritten as

$$
\left|Y_{1}(s)-Y_{1}(t)\right| \leqslant q\left\|X_{1}\right\|_{L}\|s-t\|
$$

for $s, t \in[0,1] \times S^{p-1}$. Also note that hypotheses $(a)$ and $(b)$ imply $E\left\|X_{1}\right\|_{L}^{2}<\infty$.

Next, note that the minimal number of closed balls of radius $\leqslant \epsilon$ which cover $S^{p-1}$ is of the order $c \epsilon^{1-p}$ for some constant $c$.

Then, the minimal number of such balls which cover the cylinder $[0,1] \times S^{p-1}$ is $N(\epsilon)=c \epsilon^{-p}$. If $H(\epsilon)=\lg N(\epsilon)$ denotes the metric entropy of $[0,1] \times S^{p-1}$ (corresponding to the Euclidean distance), it is easy to check that

$$
\int_{0}^{1} H^{\frac{1}{2}}(\epsilon) \mathrm{d} \epsilon<\infty
$$

Conditions (7.1) and (7.2) show that the central limit theorem of Jain \& Marcus (1975) can be applied. Thus,

$$
\sqrt{ } n\left[\sum_{i=1}^{n} j\left(X_{i}\right) / n-E j\left(X_{1}\right)\right] \rightarrow Z \quad \text { weakly, }
$$

where $Z$ is Gaussian in $C\left([0,1] \times S^{p-1}\right)$.

Since $E\left(s_{f}\right)=s_{E f}$ for any random set $f: \Omega \rightarrow \mathscr{K}_{\mathrm{e}}\left(\mathbb{R}^{p}\right)$, it is not difficult to show that $E j\left(X_{1}\right)=j\left(E X_{1}\right)$.

This fact, together with (7.3), the continuous mapping theorem, and the fact that $j$ is an isometry, implies

$$
\sqrt{ } n d_{\infty}\left(\sum_{i=1}^{n} X_{i} / n, E X_{1}\right) \rightarrow\|Z\|_{\infty} \quad \text { weakly. }
$$

(2) Assume now that $X_{n}: \Omega \rightarrow \mathscr{F}_{L}\left(\mathbb{R}^{p}\right)$, and consider the fuzzy random variables co $X_{n}$ (see §5). The Shapley-Folkman lemma (see Arrow \& Hahn 1971) gives

$$
d_{\infty}\left(\sum_{i=1}^{n} X_{i}, \operatorname{co} \sum_{i=1}^{n} X_{i}\right) \leqslant \sqrt{ } p \max _{1 \leqslant i \leqslant n}\left\|\operatorname{supp} X_{i}\right\| .
$$

Therefore, we can write:

$$
\begin{aligned}
\sqrt{ } n d_{\infty}\left(\sum_{i=1}^{n} X_{i} / n, E\left(\operatorname{co} X_{1}\right)\right) \\
\leqslant \sqrt{ } n d_{\infty}\left(\sum_{i=1}^{n} X_{i} / n, \sum_{i=1}^{n} \operatorname{co} X_{i} / n\right)+\sqrt{ } n d_{\infty}\left(\sum_{i=1}^{n} \operatorname{co} X_{i} / n, E\left(\operatorname{co} X_{1}\right)\right) .
\end{aligned}
$$

Note that if $u \in \mathscr{F}_{L}\left(\mathbb{R}^{p}\right)$, then $\|\operatorname{co} u\|_{L} \leqslant\|u\|_{L}$ so $E\left\|\operatorname{co} X_{1}\right\|_{L}^{2}<\infty$ and the second term of the above inequality converges weakly to $\|Z\|_{\infty}$ by part (1) of the proof. For the first term, we use (7.4), so that

$$
\sqrt{ } n d_{\infty}\left(\sum_{i=1}^{n} X_{i} / n, \sum_{i=1}^{n} \operatorname{co} X_{i} / n\right), \leqslant \sqrt{ } p \max _{1 \leqslant i \leqslant n}\left\|\operatorname{supp} X_{i}\right\| / \sqrt{ } n \text {. }
$$


But $E\left\|\operatorname{supp} X_{1}\right\|^{2}<\infty$ implies that $\max _{1 \leqslant i \leqslant n}\left\|\operatorname{supp} X_{i}\right\| / \sqrt{ } n \rightarrow 0$ weakly (see, for example, Breiman i 968 , p. 188). It follows, finally, that

$$
\sqrt{ } n d_{\infty}\left(\sum_{i=1}^{n} X_{i} / n, E\left(\operatorname{co} X_{1}\right)\right) \rightarrow\|Z\|_{\infty}
$$

weakly, concluding the proof.

\section{CONCLUDING REMARKS}

One of the main difficulties in proving the SLLN for fuzzy random variables was the fact that the space $\left(\mathscr{F}\left(\mathbb{R}^{p}\right), d_{\infty}\right)$ is not separable. It would be possible to overcome this, by assuming that $\left\{X_{k} \mid k \geqslant 1\right\}$ are IID fuzzy random variables which are separably-valued a.e. Such a hypothesis, however, does not seem to be natural in the context of the SLLN.

We also mention that it is possible to define a metric in $\mathscr{F}\left(\mathbb{R}^{p}\right)$, more general than $d_{1}$, by the formula

$$
d_{r}(u, v)=\left[\int_{0}^{1} d^{r}\left(L_{\alpha} u, L_{\alpha} v\right) \mathrm{d} \alpha\right]^{1 / r}, \quad r \geqslant 1 .
$$

The SLLN holds with respect to $d_{r}$ as well but for the clarity of the exposition we have chosen the metric $d_{1}$.

The central limit theorem proved in $\$ 7$ works for fuzzy random variables with values in $\mathscr{F}_{L}\left(\mathbb{R}^{p}\right)$. From the practical point of view, it might be difficult to check the Lipschitz condition for the function $\alpha \rightarrow L_{\alpha} u$.

In the following, we state some sufficient conditions which imply this Lipschitz condition.

(1) Let $u \in \mathscr{F}\left(\mathbb{R}^{p}\right)$. If there exists a constant $M>0$ such that $|u(x)-u(y)| \geqslant$ $M\|x-y\|$ for $x, y \in \operatorname{supp} u$, then $u \in \mathscr{F}_{L}\left(\mathbb{R}^{p}\right)$.

This statement can be proved by using properties of the Hausdorff distance and compactness arguments. It gives examples of fuzzy sets in $\mathscr{F}_{L}\left(\mathbb{R}^{p}\right)$ but it is quite restrictive, since $u$ satisfying the above condition must be a one-to-one function.

(2) Let $u \in \mathscr{F}\left(\mathbb{R}^{p}\right)$. If

$$
\min _{1 \leqslant j \leqslant p} \inf \left\{\left|\frac{\partial u}{\partial x_{j}}(x)\right|: x \in \operatorname{supp} u \backslash L_{1} u\right\}>0
$$

then $u \in \mathscr{F}_{L}\left(\mathbb{R}^{p}\right)$. Here the infimum is taken over all points in $\operatorname{supp} u \backslash L_{1} u$ where the derivatives of $u$ exist.

In the one dimensional case (i.e. $\mathscr{F}(\mathbb{R}))$, this condition states that if the derivative of $u$ (where it exists) is bounded away from zero on $\operatorname{supp} u \backslash L_{1} u$, then $u \in \mathscr{F}_{L}(\mathbb{R})$.

M.L.P. and D.A.R. were supported by the National Science Foundation Grant IST-7918468, and the Office of Naval Research Contract N00014-85-K-0648. 


\section{REFERENCES}

Arrow, K. J. \& Hahn, F. H. 1971 General competitive analysis. Holden-Day.

Artstein, Z. \& Vitale, R. A. 1975 A strong law of large numbers for random compact sets. Ann. Prob. 3, 879-882.

Aumann, R. J. ${ }_{1965}$ Integrals of set-valued functions. J. math. Anal. Appl. 12, 1-22.

Billingsley, P. 1968 Convergence of probability measures. Wiley.

Breiman, L. 1968 Probability. Addison-Wesley.

Chow, Y. S. \& Teicher, H. 1978 Probability theory. Springer.

Cressie, N. i 979 A central limit theorem for random sets. Z. Wahrscheinlichkeitstheor. Verw. Geb. 49, 37-47.

Debreu, G. 1967 Integration of correspondences. In Proc. Fifth Berkeley Symp. math. statist. probability, vol. II, part 1, 351-372. University of California Press (1967).

Féron, R. 1979 Ensembles aléatoires flous dont la fonction d'appartenance prend ses valeurs dans un treillis distributif fermé. In Publ. Econométriques, vol. xII, Fasc. 1, 81-118.

Giné, E., Hahn, M. G. \& Zinn, J. 1982 Limit theorems for random sets: an application of probability in Banach space results. In Proc. Fourth Int. Conf. on Probability in Banach Space (In the press.)

Jain, N. C. \& Marcus, M. B. 1975 Central limit theorem for $C(S)$-valued random variables. J. Funct. Anal. 19, 216-231.

Lowen, R. 1980 Convex fuzzy sets. Fuzzy Sets and Systems 3, 291-310.

Lyashenko, N. N. 1979 On limit theorems for sums of independent compact random subsets in the Euclidean space. Zap. Nauch. Semin., LOMI 85, 113-128. (In Russian.)

Matheron, G. 1975 Random sets and integral geometry. Wiley.

Negoita, C. V. \& Ralescu, D. A. 1975 Applications of fuzzy sets to systems analysis. Wiley.

Puri, M. L. \& Ralescu, D. A. $1983 a$ Strong law of large numbers for Banach space valued random sets. Ann. Prob. 11, 222-224.

Puri, M. L. \& Ralescu, D. A. $1988_{3} b$ Differentials of fuzzy functions. J. math. Anal. Appl. 91. $552-558$.

Puri, M. L. \& Ralescu, D. A. 1985 Limit theorems for random compact sets in Banach space. Math. Proc. Camb. Phil. Soc. 97, 151-158.

Puri, M. L. \& Ralescu, D. A. 1986 Fuzzy random variables. J. math. Anal. Appl. 114, 151-158.

Rådström, H. 1952 An embedding theorem for spaces of convex sets. Proc. Am. math. Soc. 3. 165-169.

Taylor, R. L. 1978 Stochastic convergence of weighted sums of random elements in linear spaces. (Lecture Notes in Mathematics, no. 672.) Springer.

Trader, D. A. \& Eddy, W. F. I98I A central limit theorem for the Minkowski sum of random sets. Preprint, Carnegie-Mellon University.

Vitale, R. A. 198I A central limit theorem for random convex sets. Preprint. Claremont Graduate School, Claremont, California.

Weil, W. 1982 An application of the central limit theorem for Banach space-valued random variables to the theory of random sets. Z. Wahrscheinlichkeitstheor. Verw. Geb. 60, 203-208.

Zadeh, L. A. 1965 Fuzzy sets. Inf. Control 8, 338-353. 